\title{
Tratamiento de la hiperpotasemia con diálisis peritoneal
}

\author{
Isidro Parada López, Inés Carmen de la Morena, Teresa Núñez, Ana Iglesias, Eva Campos, Antonia Viveros
}

\section{Hospital Ramón y Cajal. Madrid}

\section{Introducción:}

La hiperpotasemia es una complicación menos frecuente en diálisis peritoneal (DP) que en hemodiálisis (HD). No obstante, cuando pacientes en tratamiento con DP presentan hiperpotasemia severa (potasio $>6.5 \mathrm{mEq} / \mathrm{l}$ ), la tendencia en muchas de las unidades es tratarlas con HD de forma aguda mediante catéter temporal.

\section{Objetivo:}

Analizar nuestra experiencia en el tratamiento de hiperpotasemia severa en pacientes en programa de DP sin realizar HD aguda.

\section{Pacientes y métodos:}

Estudio retrospectivo. Desde enero de 2007 hasta febrero de 2012 se trataron en nuestra unidad ocho episodios de hiperpotasemia ( $>6.5 \mathrm{mEq} / \mathrm{l})$ presentados en seis enfermos (cinco con un único episodio y uno con tres episodios). Los enfermos eran cinco varones y una mujer, con edad media de 49 años (en un rango de 3767 años). La nefropatía de base fue: dos nefropatías intersticiales por pielonefritis crónica, una nefropatía por cambios mínimos, una nefropatía membranosa, una hialinosis focal y segmentaria asociada a VIH y una nefropatía no filiada. Ninguno era diabético. La media de tiempo en tratamiento previo con DP fue de 35.5 meses (rango de 12-70 meses). Tres pacientes se encontraban en tratamiento con diálisis peritoneal continua ambulatoria y tres con diálisis peritoneal automática. Cuidados de la enfermera: vigilar la monitorización, llevar a cabo el tratamiento IV, realizar intercambios manuales en
DP aproximadamente cada dos horas, conectar al paciente a la cicladora para la realización de la diálisis peritoneal automatizada y controlar la analítica del potasio.

\section{Resultados:}

La cifra media de potasio fue de $7.1 \mathrm{mEq} / \mathrm{l}$ (rango 6.6$7.6 \mathrm{mEq} / \mathrm{l}$ ). En todos los casos se confirmó la cifra de potasio y ningún suero estaba hemolizado. En seis de los episodios el ECG fue normal y dos de ellos presentaron ondas T picudas como única alteración. Todos los enfermos fueron ingresados en la Unidad de Agudos de Nefrología para su monitorización electrocardiográfica. Se instauró un tratamiento farmacológico mediante suero glucosado con insulina en todos ellos así como bicarbonato intravenoso en aquellos que presentaban acidosis asociada. La eliminación de potasio se realizó administrando resinas de intercambio iónico vía oral e intensificando la DP mediante cambios cortos de entre una $y$ dos horas de permanencia, a fin de realizar el mayor aclaramiento peritoneal posible de potasio sérico, hasta conseguir una disminución de la kalemia por debajo de $5.5 \mathrm{mEq} / \mathrm{l}$. La cifra media de intercambios realizados fue de seis (en un rango de cuatro a ocho).

\section{Conclusión:}

El manejo de la hiperpotasemia severa sin repercusión electrocardiográfica grave, en pacientes tratados con DP, se puede llevar a cabo sin necesidad de realizar una sesión aguda de HD. El paciente debe ser monitorizado mientras se intensifica el trata- 
miento con DP asociado al tratamiento conservador habitual. Pensamos que el trabajo de enfermería es fundamental en el tratamiento del manejo integral del paciente hiperpotasémico en DP por los cuidados específicos que este requiere.

\section{Referencias Bibliográficas}

1. Balcells, A. La clínica y el laboratorio. $15^{\mathrm{a}}$ ed. Masson-Salvat, 1991. Charytan D, Goldfarb DS. Indications for hospitalization of patients with 17 hyperkalemia. Arch Intern Med 2000; 160: 160511.

2. Greenberg, A. Hyperkalaemia: treatment options. 18 Semin. Nephrol. 1998; 18: 46-57 Kamel KS, Wei C. Controversial issues in the treatment of hyperkalaemia. 22 Nephrol. Dial Transplant 2003; 18: 2215-8. 